\title{
Dorsalis Pedis Artery as a Continuation of Peroneal Artery-Clinical and Embryological Aspects
}

\author{
Seema Sehmi
}

\begin{abstract}
Aim: To report a rare case of continuation of the peroneal artery as dorsalis pedis artery (DPA) in the foot.

Background: Peripheral arterial system of the lower limb especially the DPA is commonly used to diagnose the peripheral arterial diseases.

Case report: During the routine dissection of a formalized right lower limb of a 52-year-old male cadaver the arterial system of the lower limb was dissected and studied. The popliteal artery (PA) divided into anterior and posterior tibial arteries (PTA) at the lower border of the popliteus muscle. The peroneal artery, branch from the posterior tibial artery was found larger than usual. It ran downward laterally and after piercing the lower part of interosseous membrane continued as dorsalis pedis artery on the dorsum of the foot. The PTA had a normal course and divided distally into medial and lateral plantar arteries. However, the anterior tibial artery (ATA) was hypoplastic and terminated at the level of the talocrural joint. The arterial system of the left lower limb was found to be normal.
\end{abstract}

Conclusion: The present arterial variation around the ankle is important to the vascular and orthopedic surgeons to prevent the occurrence of any complications during arterial reconstructive surgeries.

Clinical significance: The knowledge of present variation by the vascular surgeons and radiographers help them to diagnose arterial injury for taking vascular graft and embolectomy procedures.

Keywords: Anatomical variations, Peripheral, Peroneal artery, Tibial artery

How to cite this Article: Sehmi S. Dorsalis Pedis Artery as a Continuation of Peroneal Artery-Clinical and Embryological Aspects. Curr Trends Diagn Treat 2018;2(1):44-46.

Source of support: Nil

Conflict of interest: None

\section{INTRODUCTION}

The development of the arterial system of the lower limb is very complex and is prone to anatomical variations. ${ }^{1}$

\section{Professor}

Department of Anatomy, Sri Guru Ram Das Institute of Medical Sciences and Research, Amritsar, Punjab, India

Corresponding Author: Seema Sehmi, Professor, Department of Anatomy, Sri Guru Ram Das Institute of Medical Sciences and Research, Amritsar, Punjab, India, Phone: 9914754354, e-mail: drseema16@gmail.com
The knowledge of these arterial variations are important as damage to them can be limb threatening. The DPA also known as a dorsal artery of the foot is the continuation of the ATA at the talocrural joint just distal to the inferior retinaculum. It runs towards the first intermetatarsal space and divides into the first dorsal metatarsal artery and deep plantar artery which form deep plantar arch. ${ }^{2}$ Normally, the PA is the continuation of the femoral artery. It traverses the popliteal fossa, and it descends obliquely to the distal border of the popliteal muscle. It then divides into anterior and PTA. The ATA runs to the anterior compartment of the leg through an aperture in the proximal part of the interosseous membrane and continues as dorsalis pedis artery. The peroneal artery arises about $2.5 \mathrm{~cm}$ distal to the popliteus muscle from the posterior tibial artery. Reaching the inferior tibiofibular syndesmosis, it divides into the calcaneal branches. Its perforating branch traverses the interosseous membrane about $5 \mathrm{~cm}$ proximal to the lateral malleolus to enter the extensor compartment where it anastomoses with the anterior lateral malleolar artery. ${ }^{3}$ Sometimes this arrangement can be different due to developmental diversity. Whenever either anterior or posterior tibial artery is hypoplastic, the peroneal artery enlarges and directly supplies their distal distribution areas. ${ }^{4}$ The DPA with its major branches is the preferentially used recipient vessel. The myocutaneous dorsalis pedis arterialized flap can be safely used as an island flap to cover the ankle or heel and as a free flap for palm defects. ${ }^{5}$ As variation in dorsalis pedis artery is not uncommon, it is essential to have preoperative angiography for any abnormality to prevent risks during surgical intervention.

\section{CASE REPORT}

During routine dissection for undergraduate students at Sri Guru Ram Das Medical College, Amritsar an arterial variation was found in the right lower limb of a formalin embalmed 52-year male cadaver. The usual branching of the PA into an ATA and the PTA at the lower border of the popliteus muscle was observed (Fig. 1). The PTA had a normal course, and $6 \mathrm{~cm}$ distal to the inferior border of the popliteus gave rise to the $\mathrm{PA}(3.56 \mathrm{~mm}$ in diameter) and distally divided into medial and lateral plantar arteries (Fig. 2). The ATA was hypoplastic $(1.6 \mathrm{~mm})$, pierced the interosseous membrane and descended deep to the extensor digitorum 


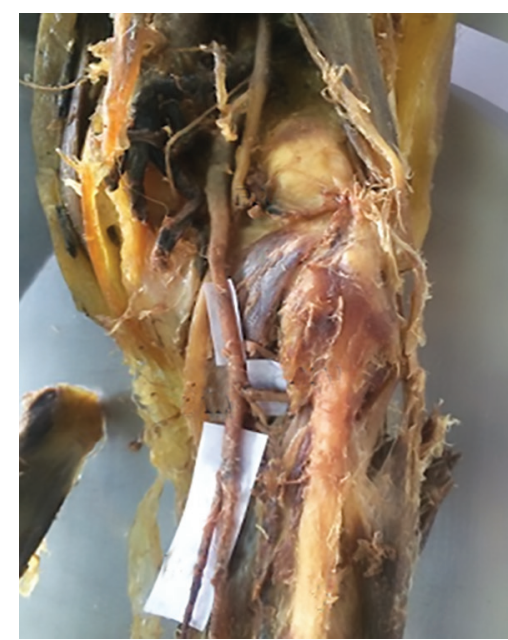

Fig. 1: Right view of popliteal fossa showing popliteal artery (PA) dividing into anterior tibial artery (ATA) and posterior tibial artery (PTA) with posterior tibial artery (PTA) giving rise to peroneal artery (PA)

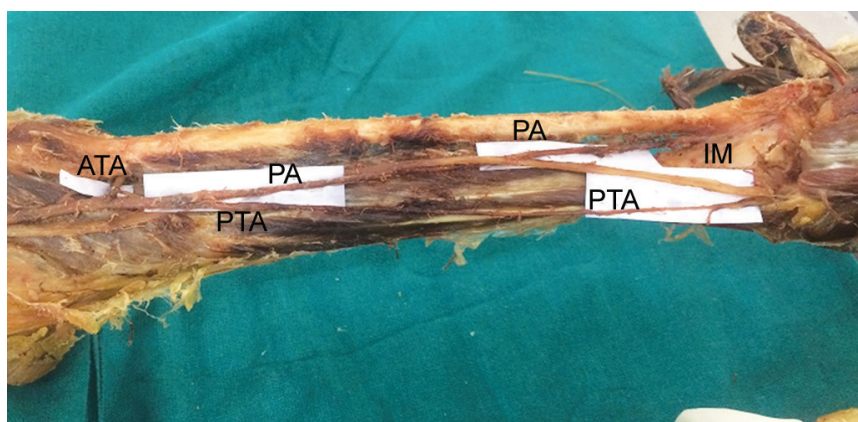

Fig. 2: Posterior view of right leg showing peroneal artery (PA) piercing interosseous membrane (IM). Posterior tibial artery (PTA).

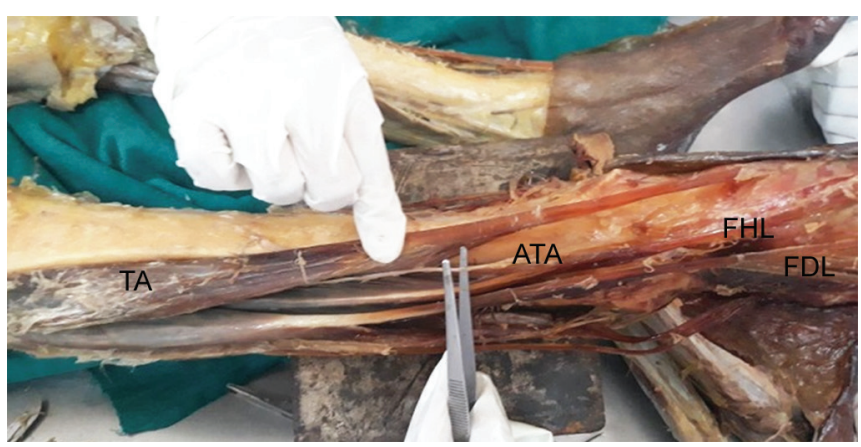

Fig. 3: Anterolateral view of right leg showing anterior tibial artery (ATA) terminating as a minute twig at the ankle joint. TA, tibialis anterior; flexor hallucis longus (FHL); flexor digitorum longus (FDL)

longus where it ended $6 \mathrm{~cm}$ above the ankle-joint $(0.14 \mathrm{~mm})$ (Fig. 3). The peroneal artery pierced the lowest portion of the interosseous membrane and continued as DPA (Fig. 4).

\section{DISCUSSION}

In the present case, the PTA was of normal size but the ATA was hypoplastic. The peroneal artery was larger than usual and crossed the lowest portion of the interosseous membrane and continued as DPA.

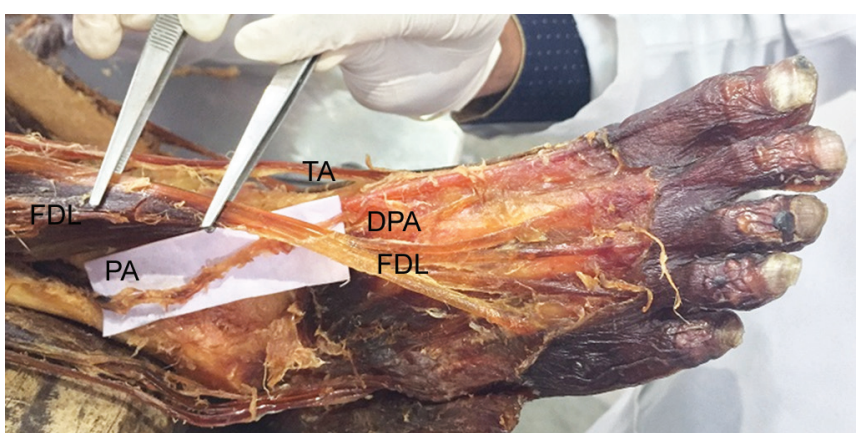

Fig. 4: Anterior view of right foot showing Peroneal artery (PA) after piercing the interosseous membrane continuing as Dorsalis Paedis artery (DPA) between Tibialis anterior (TA) and Flexor digitorum longus (FDL).

Variations in the branching pattern of the PA and arterial supply of the foot are classified as follows: ${ }^{6}$

- Normal level of PA branching

- High division of PA

- Hypoplastic or Aplastic branching with altered distal supply. This is further divided into three subtypes:

1. Hypoplastic or Aplastic PTA-distal PTA replaces the peroneal artery.

2. Hypoplastic or Aplastic ATA- dorsalis pedis artery replaces the peroneal artery.

3. Hypoplastic or Aplastic PTA or ATA. Plantar arteries and DPA replace the fibular artery.

The present case belongs to Type $3 \mathrm{~b}$ of variation in the branching pattern of the PA. In $8 \%$ of cases the DPA arose as a continuation of the perforating branch of peroneal artery. ${ }^{7}$ Similarly, Shetty et al. ${ }^{8}$ reported a rare case in which the ATA was hypoplastic and peroneal artery continued as DPA. Hypoplasia of the ATA is found to be associated with severe bony malformation of the leg ${ }^{9}$ or with campomelic syndrome. ${ }^{10}$ In the present case, the right DPA was replaced by the peroneal artery but no bony malformation was detected Similar variation was described unilaterally by Ali and Mohajir ${ }^{11}$ and bilaterally by Tuncel et al. ${ }^{12}$ The anatomical variations of the arterial system of lower limb are important determinants of femorodistal, popliteal as well as tibial arterial reconstructions. The presence, as well as the luminal diameter of the ATA, is important for anterior femorotibial grafts. ${ }^{13}$ Hypoplastic PTA if the perforating branch of fibular artery replaces present and harvesting the fibula along with the fibular artery can endanger the nutrition of the foot. ${ }^{6}$

\section{Embryology}

The axial artery of the lower limb arising from the dorsal root of the umbilical artery forms the primordial central artery of the lower limb. The femoral artery gradually increases in size, and coincidently the axial artery starts disappearing. Initially, the axial artery passes deep to the PA as a deep PA. 
At the proximal end of the popliteus muscle, the axial artery gives rise to the primitive PTA and primitive fibular artery and both run on the dorsal surface of the popliteus muscle. These two arteries fuse with the axial artery proximal to distal up to the inferior margin of the popliteus muscle known as a superficial popliteal artery which is the definitive part of the popliteal artery. A communicating branch joins the superficial PA to the distal part of the deep PA which later on forms ATA. Also, most of the remainder of the deep PA disappear. ${ }^{2,14,15}$ Thus the arterial variations of the lower limb can be explained by the combination of persistent primitive arterial segments, abnormal fusions and hypoplasia or absence of a particular segment. ${ }^{16}$

\section{CONCLUSION}

Variations of the dorsalis pedis artery are clinically important as it is used to record peripheral arterial pulsations, in microvascular surgeries of the foot during replantation, reconstruction and repair and use of musculocutaneous flaps in operating nonhealing diabetic foot ulcers. It is advised to do preoperative angiography to diagnose the anatomical variations in the course and distribution of ATA or dorsalis pedis artery.

\section{REFERENCES}

1. Sahin B and Bilgic S. Two rare arterial variations of the deep femoral artery in the new born. Surg Radial Anat. 1998;20:233-235.

2. Standring S. Gray's Anatomy the Anatomical Basis of Clinical Practice. 41st Ed, London, Elsevier. 2016;1405-1415.
3. Lippert H, Pabst R. Popliteal artery. Arterial Variations in Man [Internet]. J.F. Bergmann-Verlag; 1985;62-62.

4. Chester B, Anson M, McVay. Surgical Anatomy. W.B.Saunders Company, Japan, 1985.

5. Aithal PA, Patil J, D'Souza MR, Kumar N, Nayak BS, Guru A. Anomalous origin of dorsalis pedis artery and its clinical significance. Chrismed J Health Res. 2015;2:302-304.

6. Kim D, Orron DE, Skillman J J. Surgical significance of popliteal arterial variants. A unified angiographic classification. Ann Surg. 1989;210(6):776-781.

7. Maral TM and Celik TC. Anomalous dorsalis pedis artery. Surg Radiol Anat. 1994;6:319-323.

8. Shetty SD, Nayak S, Kumar N, Abhintha P. Hypoplastic anterior tibial artery associated with continuation of fibular (peroneal) artery as dorsalis pedis artery. A case report. Int J Morphol. 2013;31:136-139.

9. Hootnick DR, Packard DS, Levinsohn EM, Lebowitz MR, Lubicky JP. The anatomy of a congenitally short limb with clubfoot and ectrodactyly. Teratology. 1984;29(2):155-164.

10. Rodríguez JI. Vascular anomalies in campomelic syndrome. Am J Med Genet. 1993;46 (2):185-192.

11. Ali M. Dorsalis pedis artery-Variation and clinical significance. J Ind Med Assoc. 1996;94:417-418.

12. Tuncel M, Maral T, Celik H, Tasçioglu B. A case of bilateral anomalous origin for dorsalis pedis arteries (anomalous dorsalis pedis arteries). Surg Radiol Anat. 1994;16:319-323.

13. Plecha EL, Seabgook GR, Bandyk DF, Towne JB. Determinants of successful peroneal artery bypass. J Vasc Surg. 1993; 17: 97-105.

14. Senior HD. The development of the arteries of the human lower extremity. Am J Anat. 1919;25(1):54-95.

15. Kesavi D, Singh K, Melani RS. Anomalous course of dorsalis pedis artery. Anatomical adjuncts. 2002;3:29-31.

16. Atanasova M, Georgiev GP, Jelev L. Intriguing variations of the tibial arteries and their clinical implications. Int J Anat Var. 2011;4:45-47. 